\title{
CONVOLUTION OPERATORS ASSOCIATED WITH VECTOR MEASURES
}

\author{
by MANGATIANA A. ROBDERA and PAULETTE SAAB
}

(Received 6 January, 1997)

\begin{abstract}
In this note, we present a thorough investigation of convolution operators that are naturally associated to vector measures. We characterize those convolution operators that are weakly compact and compact on $\mathcal{L}^{1}(G)$ and $\mathcal{C}(G)$ as well as those that are $p$ summing, $(1 \leq p \leq \infty)$ and nuclear on $\mathcal{C}(G)$.
\end{abstract}

1. Preliminaries. Let $G$ be a compact metrizable abelian group with $\lambda$ a normalized Haar measure and let $X$ be a Banach space. For $1 \leq p<\infty$, let $\mathcal{L}^{P}(G, X)\left(\right.$ resp. $\left.\mathcal{L}^{\infty}(G, X)\right)$ denote the Banach space of (all classes of) $\lambda$-Bochner $p$-integrable (resp. (all classes of) $\lambda$ measurable functions that are essentially bounded) $X$-valued functions defined on $G$. We also let $\mathcal{C}(G, X)$ denote the Banach space of all continuous $X$-valued functions defined on $G$. If $X$ is the field of scalars, we simply write $\mathcal{L}^{P}(G)$ (resp. $\mathcal{L}^{\infty}(G)$ ) and $\mathcal{C}(G)$. For an $X$-valued measure of bounded variation $\mu$, the formula $f * \mu(t)=\int_{G} f(t-s) d \mu(s)$ defines a bounded linear operator $T_{\mu}: \mathcal{L}^{1}(G) \rightarrow \mathcal{L}^{1}(G, X)$ (resp. $T_{\mu}: \mathcal{C}(G) \rightarrow \mathcal{C}(G, X)$ ) by $T_{\mu}(f)=f * \mu$ for every $f \in \mathcal{L}^{1}(G)$ (resp. $\mathcal{C}(G)$ ). Such an operator $T_{\mu}$ is called a convolution operator associated to the measure $\mu$.

For the case where $X$ is reduced to the scalars, convolution operators associated to regular Borel scalar measures on compact groups have been well studied and understood. For example, one has the following theorem:

THEOREM 1.1. Let $G$ be a compact metrizable abelian group with Haar measure $\lambda$. Let $\mu$ be a regular Borel scalar measure on $G$. Let $T_{\mu}: \mathcal{L}^{1}(G) \rightarrow \mathcal{L}^{1}(G)$ be the convolution defined by $T_{\mu}(f)=f * \mu$. Each of the following statements implies all the others:

(a) the operator $T_{\mu}$ is compact;

(b) the operator $T_{\mu}$ is weakly compact;

(c) the operator $T_{\mu}$ is representable;

(d) the measure $\mu$ is $\lambda$-continuous.

Proofs of these results can be found in [4], [8] and [11] among others. We simply notice that the implication (b) $\Rightarrow$ (c) follows from the fact that every weakly compact operator on $\mathcal{L}^{2}(G)$ is representable; this is of course due to N. Dunford and B. J. Pettis. The equivalence (c) $\Longleftrightarrow(d)$ is due to A. Costé, a proof can be found in [4, p. 90-91]. The implication (d) $\Rightarrow$ (a) is a simple exercise and a proof can be inferred from the vector valued case which will be treated in Section 2. We will see that these equivalences remain true for the vector valued case.

Moreover, each one of the assertions of Theorem 1.1 is equivalent to every one of the following theorem.

Glasgow Math. J. 40 (1998) 367-384. 
THEOREM 1.2. Let $G$ be a compact metrizable abelian group with Haar measure $\lambda$. Let $\mu$ be a regular Borel scalar measure on $G$. Let $T_{\mu}: \mathcal{C}(G) \rightarrow \mathcal{C}(G)$ be the convolution defined by $T_{\mu}(f)=f * \mu$. Then the following assertions are equivalent:

$\left(\mathrm{a}^{\prime}\right)$ the operator $T_{\mu}: \mathcal{C}(G) \rightarrow \mathcal{C}(G)$ is compact;

(b') the operator $T_{\mu}: \mathcal{C}(G) \rightarrow \mathcal{C}(G)$ is weakly compact;

$\left(\mathrm{c}^{\prime}\right)$ the measure $\mu$ is $\lambda$-continuous.

Proof. $\left(\mathrm{a}^{\prime}\right) \Rightarrow\left(\mathrm{b}^{\prime}\right)$ is of course obvious. The implication $\left(\mathrm{b}^{\prime}\right) \Rightarrow\left(\mathrm{c}^{\prime}\right)$ follows from a result of $[6$, p. 278] and a proof can be inferred from the vector valued case which will be treated in Section 3.

The implication $\left(\mathrm{c}^{\prime}\right) \Rightarrow\left(\mathrm{a}^{\prime}\right)$ follows from the fact that trigonometric polynomials are dense in $\mathcal{L}^{1}(G)$. To see this, note that if $\mu=f \cdot \lambda$, where $f \in \mathcal{L}^{1}(G)$, then these exists a sequence $\left\{p_{n}\right\}$ of trigonometric polynomials such that

$$
\lim _{n \rightarrow \infty}\left\|f-p_{n}\right\|_{1}=0 .
$$

Since $\left\|T_{\mu}-T_{p_{n}}\right\| \leq\left\|f-p_{n}\right\|_{1}$, and since each $T_{p_{n}}$ is a finite rank operator, it follows that $T_{\mu}$ is a compact operator.

More details and related results of this theorem can be seen in [8] and [11]. Unlike the case of convolution operators on $\mathcal{L}^{1}(G)$, we will see in Section 3 that the results of Theorem 1.2 do not hold for their vector valued counterparts. Also in Section 3 we shall give a complete answer to the following natural question.

Question 1. For what Banach space $X$ is the following true?

Given a compact metrizable abelian group $G$ and an $X$-valued measure of bounded variation $\mu$ on $G$, then the convolution operator $T_{\mu}: \mathcal{C}(G) \rightarrow \mathcal{C}(G, X)$ is compact whenever $T_{\mu}$ is weakly compact.

Finally, we shall study in Section 4 the extension to the vector valued case of the following result.

THEOREM 1.3. Let $G$ be a compact metrizable abelian group with Haar measure $\lambda$. Let $\mu$ be a regular Borel scalar measure on $G$. Let $T_{\mu}: \mathcal{C}(G) \rightarrow \mathcal{C}(G)$ be the convolution defined by $T_{\mu}(f)=f * \mu$. Then

(a) the operator $T_{\mu}$ is l-summing if and only if $\mu=\phi \cdot \lambda$ where $\phi \in \mathcal{L}^{\infty}(G)$;

(b) the operator $T_{\mu}$ is nuclear if and only if $\mu=\phi \cdot \lambda$ where $\phi \in \mathcal{C}(G)$.

First, let us fix some notations and terminologies.

Notations 1. If $X$ and $Y$ are Banach spaces, a bounded linear operator $T: X \rightarrow Y$ is compact (resp. weakly compact), if $T$ maps the unit ball of $X$ into a relatively compact (resp. a relatively weakly compact) subset of $Y$.

If $G$ is a compact metrizable abelian group, we will denote by $\hat{G}$ its dual group and by $\lambda$ a normalized Haar measure on $G$. If $X$ is a Banach space and $f \in \mathcal{L}^{1}(G, X)$, we denote by $\hat{f}$ its Fourier transform, defined as follows:

$$
\hat{f}(\gamma)=\int_{G} f(t) \bar{\gamma}(t) d \lambda(t), \text { for each } \gamma \in \hat{G}
$$


More generally, if $\mu$ is an $X$-valued vector measure of bounded variation on $G$, we denote by $\hat{\mu}$ its Fourier transform defined as follows:

$$
\hat{\mu}(\gamma)=\int_{G} \bar{\gamma} d \mu, \text { for each } \gamma \in \hat{G} .
$$

Here one should note that when $f \in \mathcal{L}^{\prime}(G, X)$ and or more generally, when $\mu$ is an $X$-valued measure, $\hat{f}(\gamma)$ and $\hat{\mu}(\gamma)$ are elements of $X$ for each $\gamma$ in $\hat{G}$. Also if $f \in \mathcal{L}^{1}(G)$, and if $t \in G$, we denote by $f$, the element of $\mathcal{L}^{\prime}(G)$ defined by

$$
f_{l}(x)=f(x-t), \text { for each } x \in G .
$$

The $\sigma$-field of Borel subsets of $G$ will be denoted by $\mathcal{B}(G)$. If $A$ is a Borel subset of $G$, then $\chi_{A}$ will stand for the characteristic function of $A$, i.e.

$$
\chi_{A}(x)= \begin{cases}1 & \text { if } x \in A \\ 0 & \text { if } x \notin A\end{cases}
$$

If $X$ is a Banach space and $\mu$ is an $X$-valued measure on $G$ that is of bounded variation, we say that $\mu$ has relatively compact range if $\{\mu(A): A \in \mathcal{B}(G)\}$ is a relatively compact subset of $X$.

Finally, all notions not defined can be found in [3], [4] or [11].

2. Convolution operators on $\mathcal{L}^{1}(G)$. Our first result extends the assertions of Theorem 1.1 to the vector case.

Theorem 2.1. Let $G$ be a compact metrizable abelian group with Haar measure $\lambda$. Let $X$ be a Banach space and let $\mu$ be an $X$-valued measure of bounded variation on $G$. Let $T_{\mu}: \mathcal{L}^{1}(G) \rightarrow \mathcal{L}^{1}(G, X)$ be the convolution defined by $T_{\mu}(f)=f * \mu$ for all $f \in \mathcal{L}^{1}(G)$. Then the following properties are equivalent:

(a) the operator $T_{\mu}$ is compact;

(b) the operator $T_{\mu}$ is weakly compact;

(c) the operator $T_{\mu}$ is representable;

(d) the measure $\mu=h \cdot \lambda$, where $h \in \mathcal{L}^{l}(G, X)$.

Proof. (a) $\Rightarrow$ (b) $\Rightarrow$ (c) are immediate.

(c) $\Rightarrow$ (d). The following argument is due to A. Coste $([4$, p. 90-91]) for the corresponding scalar case, we shall include his argument which extends to the vector case for the sake of completeness. Suppose that the convolution operator $T_{\mu}$ is representable and let $\psi$ be the element of $\mathcal{L}^{\infty}\left(G, \mathcal{L}^{\prime}(G, X)\right)$ such that $T_{\mu}(f)(\cdot)=\int_{G} f(s) \psi(s)(\cdot) d \lambda(s)$, for every $f \in \mathcal{L}^{1}(G)$. The function $\psi$ can be considered as function of two variables in the following manner:

$$
\phi(u, v)=\psi(u)(v)
$$

for $(u, v) \in G \times G$. 
Define $h \in \mathcal{L}^{l}(G, X)$ by $h(t)=\int_{G} \phi(u-t, u) d \lambda(u)$. For each $f \in \mathcal{L}^{l}(G)$, a simple change of variable in tandem with Fubini's Theorem establishes the following:

$$
\begin{aligned}
f * h(s) & =\int_{G} f(t) h(s-t) d \lambda(t) \\
& =\int_{G} f(t) \int_{G} \phi(u+t-s, u) d \lambda(u) d \lambda(t) \\
& =\int_{G} \int_{G} f(t) \phi(v+t, v+s) d \lambda(v) d \lambda(t) \\
& \left.=\iint_{G} f(t) \phi(v+t, v+s) d \lambda(t)\right) d \lambda(v)
\end{aligned}
$$

Again for each $v \in G$ and each $t \in G$, if one denotes $f_{v}(t)=f(t-v)$, then one notices that

$$
\begin{aligned}
\int_{G \times G} f(t) \phi(v+t, v+s) d \lambda(t) d \lambda(v) & =\int_{G} \int_{G} f_{v}(t+v) \phi(v+t, v+s) d \lambda(t) d \lambda(v) \\
& =\int_{G} T_{\mu}\left(f_{v}\right)(v+s) d \lambda(v) \\
& =\int_{G} f_{v} * \mu(v+s) d \lambda(v) \\
& =\int_{G} \int_{G} f_{v}(v+s-t) d \mu(t) d \lambda(v) \\
& =\int_{G} \int_{G} f(s-t) d \mu(t) d \lambda(v) \\
& =\int_{G} f * \mu(s) d \lambda(v)=f * \mu(s),
\end{aligned}
$$

it then follows that $f * h=f * \mu$, for each $f \in \mathcal{L}^{1}(G)$. This shows that $\mu=h \cdot \lambda$.

(d) $\Rightarrow$ (a) Suppose $\mu=g \cdot \lambda$, where $g \in \mathcal{L}^{1}(G, X)$. Let $\left(i_{n}\right)_{n \in \mathbb{N}}$ be a good approximate identity on the group $G$ [6]. Consider the sequence of convolution operators $\left(T_{i_{n}}\right)$ associated to the sequence of functions $\left\{i_{n}\right\}$, that is $T_{i_{n}}(f)=f * i_{n}$, for each $f \in \mathcal{L}^{1}(G)$, where $f * i_{n}(t)=$ $\int_{G} f(t-s) i_{n}(s) d \lambda(s)$, for each $f \in \mathcal{L}^{1}(G)$. By Theorem 1.1, since the $i_{n}$ are in $\mathcal{L}^{1}(G)$ the operators $T_{i_{n}}$ are all compact. It follows that for each $n \geq 1$ the operator $T_{\mu} T_{i_{n}}$ is also compact. Since for every $f \in \mathcal{L}^{l}(G)$, we have

$$
\left\|T_{\mu} T_{i_{n}}(f)-T_{\mu}(f)\right\|=\left\|f * i_{n} * g-f * g\right\| \leq\|f\|_{1}\left\|i_{n} * g-g\right\|_{1},
$$


and since it is easily seen that the sequence $\left(i_{n} * g\right)$ converges to $g$ in $\mathcal{L}^{1}(G, X)[7]$, the inequality (2.3) then implies that the sequence $\left\{T_{\mu} T_{i_{n}}\right\}$ converges to the operator $T_{\mu}$ in the operator norm and hence it shows that the operator $T_{\mu}$ is compact.

As a consequence, this result sets up the following characterization of Banach spaces with the Radon-Nikodym property (RNP); for more details on the RNP see [4].

Corollary 2.2. Let $G$ be a compact metrizable abelian group with Haar measure $\lambda$. A Banach space $X$ has $R N P$ if and only if for every $\lambda$-continuous $X$-valued countably additive measure of bounded variation $\mu$ on $G$, the convolution operator $T_{\mu}: \mathcal{L}^{1}(G) \rightarrow \mathcal{L}^{1}(G, X)$ defined by $T_{\mu}(f)=f * \mu$ for all $f \in \mathcal{L}^{\mathcal{1}}(G)$ is compact.

3. Compact and weakly compact convolution operators on $\mathcal{C}(\mathbf{G})$. The study of convolution operators associated to vector measure on $\mathcal{C}(G)$ spaces turned out to be very interesting. Unlike their behavior on $\mathcal{L}^{1}(G)$ spaces where results in the scalar case easily extended to the vector case, the analogue of Theorem 1.2 does not extend to the vector case. In what follows, we present two easy examples that illustrate such failure.

EXAMPLE 1 . Let $\mathbb{T}$ be the circle group. Let $\mu$ be the $c_{0}$-valued measure defined by

$$
\mu(A)=\left(\frac{1}{2 \pi} \int_{\mathbb{T}} \chi_{A}(s) e^{-i n s} d s\right)_{n \geq 1,}
$$

for every Borel subset $A$ of $\mathbb{T}$. Then the convolution operator $T_{\mu}$ associated to the measure $\mu$ is a weakly compact operator on $\mathcal{C}(\mathbb{T})$ that is not compact.

Proof. To see that the convolution operator $T_{\mu}$ is weakly compact on $\mathcal{C}(\mathbb{T})$, we first consider $T_{\mu}$ as a convolution operator on $\mathcal{L}^{\prime}(\mathbb{T})$. As such $T_{\mu}$ a priori takes its values in $\mathcal{L}^{1}\left(\mathbb{T}, c_{0}\right)$. We claim that $T_{\mu}$ as actually a bounded linear operator from $\mathcal{L}^{1}(\mathbb{T})$ into $\mathcal{C}\left(\mathbb{T}, c_{0}\right)$. This will show that $T_{\mu}$ as a convolution operator on $\mathcal{C}(\mathbb{T})$, can be factored through $\mathcal{L}^{1}(\mathbb{T})$, and hence it is weakly compact, since the canonical injection from $\mathcal{C}(\mathbb{T})$ into $\mathcal{L}^{\prime}(\mathbb{T})$ is weakly compact [3].

To justify the claim, notice that for $f \in \mathcal{L}^{1}(\mathbb{T})$, and for each $t \in \mathbb{T}$, one has

$$
\begin{aligned}
f * \mu(t) & =\int_{\mathbf{T}} f(t-s) d \mu(s) \\
& =\left(\frac{1}{2 \pi} \int_{\mathbb{T}} f(t-s) e^{-i n s} d s\right)_{n \geq 1} \\
& =\left(\frac{1}{2 \pi} \int_{\mathbb{T}} \bar{f}_{t}(s) \mathrm{e}^{-i n s} d s\right)_{n \geq 1} \\
& =\left(\hat{\bar{f}}_{t}(n)\right)_{n \geq 1},
\end{aligned}
$$


where $\bar{f}(s)=f(-s)$ for every $s \in \mathbb{T}$. It is a well known fact [7] and easily seen that the map $t \mapsto f_{t}$, and hence the map $t \mapsto \bar{f}_{t}$ is continuous from the group $\mathbb{T}$ into $\mathcal{L}^{1}(\mathbb{T})$. On the other hand, since $\sup _{n}|\hat{f}(n)| \leq\|f\|_{1}$ for every $f \in \mathcal{L}^{\mathcal{l}}(\mathbb{U})$, the Fourier transform $\widehat{(\cdot)}$ considered as a linear operator from $\mathcal{L}^{1}(\mathbb{T})$ into $c_{0}$ is bounded. Hence, (3.1) shows that the map $t \mapsto f * \mu(t)$ is continuous, hence $f * \mu \in \mathcal{C}\left(\mathbb{T}, c_{0}\right)$ for each $f \in \mathcal{L}^{1}(\mathbb{T})$. It also follows from (3.1) that

$$
\|f * \mu\|_{\mathcal{C}\left(\mathbb{\mathbb { N }}, c_{0}\right)}=\sup _{t \in \mathbb{\mathbb { T }}} \sup _{n}\left|\hat{\bar{f}}_{t}(n)\right|=\sup _{t \in \mathbb{T}} \sup _{n}\left|\hat{f}_{t}(n)\right| \leq\left\|f_{t}\right\|_{1}=\|f\|_{1} .
$$

This proves our claim.

On the other hand, if the convolution operator $T_{\mu}$ were compact as an operator from $\mathcal{C}(\mathbb{T})$ into $\mathcal{C}\left(\mathbb{T}, c_{0}\right)$, then so would the operator $S=\delta_{0} T_{\mu}$, where $\delta_{0}: \mathcal{C}\left(\mathbb{T}, c_{0}\right) \rightarrow c_{0}$ is the evaluation operator at 0 , i.e. $\delta_{0}(f)=f(0)$, for every $f \in \mathcal{C}\left(\mathbb{T}, c_{0}\right)$. This cannot be the case since $S$ is nothing but

$$
\begin{aligned}
S: \mathcal{C}(\mathbb{T}) & \rightarrow c_{0} \\
f & \mapsto(\hat{\bar{f}}(n))_{n>1} .
\end{aligned}
$$

$S$ is not compact: indeed the characters form a bounded set in $\mathcal{C}(\mathbb{T})$, but clearly, for any two different positive integers $n$ and $n^{\prime}$, a simple computation shows that

$$
\left\|S\left(e^{i n(\cdot)}\right)-S\left(e^{i n^{\prime}(\cdot)}\right)\right\|=1 .
$$

This finishes the proof.

EXAMPLE 2. Let $G$ be a compact metrizable abelian group with Haar measure $\lambda$, and let $\mu$ be the $\mathcal{L}^{1}(G)$-valued measure defined by

$$
\mu(A)=\chi_{A},
$$

for every Borel subset $A$ of $G$. Then the convolution operator $T_{\mu}$ associated to the measure $\mu$ is a non compact weakly compact operator on $\mathcal{C}(G)$.

Proof. As in the proof of the above example, we show that the convolution operator $T_{\mu}: \mathcal{C}(G) \rightarrow \mathcal{C}\left(G, \mathcal{L}^{\mathbf{l}}(G)\right)$ factors through $\mathcal{L}^{\prime}(G)$. It is clear that the measure $\mu$ defines a convolution operator from $\mathcal{L}^{1}(G)$ into $\mathcal{L}^{1}\left(G, \mathcal{L}^{1}(G)\right)$, still denoted by $T_{\mu}$. For each $f \in \mathcal{L}^{1}(G)$, and for each $t \in \mathcal{L}^{1}(G)$, we have

$$
f * \mu(t)=\int_{G} f(t-s) d \mu(s)=\int_{G} \bar{f}_{t}(s) d \mu(s)=\bar{f}_{l},
$$

where $\bar{f}$ is the function defined by $\bar{f}(s)=f(-s)$, for every $s \in G$. The continuity of the map $t \mapsto \bar{f}_{t}=f * \mu(t)$ shows that $f * \mu$ is indeed in $\mathcal{C}\left(G, \mathcal{L}^{1}(G)\right)$. That is the range of $T_{\mu}$ considered as a convolution operator from $\mathcal{L}^{1}(G)$ into $\mathcal{L}^{1}\left(G, \mathcal{L}^{1}(G)\right)$ is contained in $\mathcal{C}\left(G, \mathcal{L}^{1}(G)\right)$. We also have for each $f \in \mathcal{C}(G)$

$$
\begin{aligned}
\|f * \mu\|_{\mathcal{C}\left(G, \mathcal{L}^{\prime}(G)\right)} & =\sup _{t \in G}\|f * \mu(t)\|_{1} \\
& =\sup _{t \in G}\left\|\bar{f}_{t}\right\|=\|f\|_{1} .
\end{aligned}
$$


This shows that $T_{\mu}: \mathcal{C}(G) \rightarrow \mathcal{C}\left(G, \mathcal{L}^{\prime}(G)\right)$ factors through $\mathcal{L}^{\prime}(G)$, and hence it is weakly compact [3].

On the other hand, if $T_{\mu}: \mathcal{C}(G) \rightarrow \mathcal{C}\left(G, \mathcal{L}^{1}(G)\right)$ were compact, then so would the operator $S=\delta_{0} T_{\mu}: \mathcal{C}(G) \rightarrow \mathcal{L}^{1}(G)$ defined by $S f=f * \mu(0)=\bar{f}$. The operator $S$ is not compact since it is clearly seen to be a bounded linear operator from $\mathcal{C}(G)$ onto $\mathcal{C}(G)$. The proof is complete.

The next result characterizes those convolution operators that are weakly compact on $\mathcal{C}(G)$. Before we state the result, we need the following lemma:

Lemma 3.1. Let $G$ be a compact metrizable abelian group with Haar measure $\lambda$. Let $X$ be a Banach space, and let $\mu$ be an $X$-valued countably additive measure of bounded variation defined on the group $G$. Let $T_{\mu}: \mathcal{C}(G) \rightarrow \mathcal{C}(G, X)$ be the convolution operator defined by $T_{\mu}(f)=f * \mu$ for all $f \in \mathcal{C}(G)$ and let $\mathfrak{F}$ be its representing measure. Then $\mathfrak{F}$ is given by $\mathfrak{\mho}(A)=\chi_{A} * \mu$, for every $A \in \mathcal{B}(G)$.

Proof. We first notice that since the group $G$ is compact and metrizable, the class of Baire subsets and the class of Borel subsets of $G$ coincide. Thus we are done if we prove that $\mathfrak{F}(A)=\chi_{A} * \mu$, for every compact $G_{\delta}$ subset $A$ of $G$.

To see this, let $A$ be a compact $G_{\delta}$ subset of the group $G$. Let $\left(\varphi_{n}\right)$ be a sequence of continuous functions on $G$ converging pointwise to $\chi_{A}$ and such that $0 \leq \varphi_{n} \leq 1$. For each $t \in G$, one can appeal to the Lebesgue Dominated Convergence Theorem to get

$$
\begin{aligned}
\lim _{n} \varphi_{n} * \mu(t) & =\lim _{n} \int_{G} \varphi_{n}(t-y) d \mu(y) \\
& =\int_{G} \chi_{A}(t-y) d \mu(y) \\
& =\chi_{A} * \mu(t) .
\end{aligned}
$$

Now let $v$ be in the dual of $\mathcal{C}(G, X)$. Then $v$ can be considered as an $X^{*}$-valued vector measure of bounded variation (see $\left[4\right.$, p. 182]). Then there exists a function $g: G \rightarrow X^{*}$ having the following properties (see [2]):

1. $\|g(t)\|=1|v|$-almost everywhere;

2. $\int_{G} f d v=\int_{G}\langle g(t), f(t)\rangle d|v|(t)$, for $f \in \mathcal{L}^{\infty}(\mathcal{G}, \mathcal{X})$.

Thus since for each $n, \varphi_{n} * \mu$ belongs to $\mathcal{C}(G, X)$, we have

$$
\int_{G} \varphi_{n} * \mu d v=\int_{G}\left\langle g(t), \varphi_{n} * \mu(t)\right\rangle d|\nu|(t) .
$$

We also notice that

$$
\left|\left\langle g(t), \varphi_{n} * \mu(t)\right\rangle\right| \leq\|g(t)\|\left\|\varphi_{n} * \mu(t)\right\| \leq\|g(t)\|\left\|\varphi_{n} * \mu\right\|_{\infty} \leq\|\mu\| .
$$


Therefore, one can once again appeal to the Lebesgue Dominated Convergence Theorem to see that

$$
\begin{aligned}
\lim _{n} \int_{G} \varphi_{n} * \mu d \nu & =\lim _{n} \int_{G}\left\langle g(t), \varphi_{n} * \mu(t)\right\rangle d|\nu|(t) \\
& =\int_{G}\left\langle g(t), \chi_{A} * \mu(t)\right\rangle d|v|(t) \\
& =\int_{G} \chi_{A} * \mu d \nu .
\end{aligned}
$$

On the other hand, one notices that for each $v$ in the dual of $\mathcal{C}(G, X)$,

$$
\begin{array}{r}
\langle\mathfrak{F}(A), v\rangle=\left\langle T_{\mu}^{* *}\left(\chi_{A}\right), v\right\rangle \\
=\lim _{n}\left\langle T_{\mu}\left(\varphi_{n}\right), v\right\rangle \\
=\lim _{n} \int_{G} \varphi_{n} * \mu d v .
\end{array}
$$

Hence we see that $\mathfrak{\wp}(A)=\chi_{A} * \mu$ as desired. This completes the proof.

We are now ready to characterize those convolution operators that are weakly compact on $\mathcal{C}(G)$.

THEOREM 3.2. Let $G$ be a compact metrizable abelian group with Haar measure $\lambda$. Let $X$ be a Banach space, and let $\mu$ be an $X$-valued countably additive measure of bounded variation. Let $T_{\mu}: \mathcal{C}(G) \rightarrow \mathcal{C}(G, X)$ be the convolution operator defined by $T_{\mu}(f)=f * \mu$ for all $\in \mathcal{C}(G)$. Then the following properties are equivalent:

(a) the operator $T_{\mu}$ is weakly compact;

(b) the measure $\mu$ is absolutely continuous with respect to $\lambda$.

Proof. Suppose the convolution operator $T_{\mu}$ is weakly compact. We first notice that by Lemma 3.1, the representing measure $\mathfrak{\mho}$ of $T_{\mu}$ is given by $\widetilde{\mho}(A)=\chi_{A} * \mu$. Since $T_{\mu}$ is weakly compact, it follows that $\chi_{A} * \mu \in \mathcal{C}(G, X)$ for each Borel subset $A$. Therefore, for every character $\gamma$ on the group $G$, one has

$$
\begin{aligned}
\widehat{\mathscr{F}(A})(\gamma)=\widehat{\chi_{A} * \mu} \mu(\gamma)=\int_{G} \chi_{A} * \mu(t) \bar{\gamma}(t) d \lambda(t) & =\iint_{G} \chi_{A}(t-s) \bar{\gamma}(t) d \mu(s) d \lambda(t) \\
& =\iint_{G} \chi_{A}(u) \bar{\gamma}(s+u) d \lambda(u) d \mu(s) \\
& =\left(\int_{G} \chi_{A}(u) \bar{\gamma}(u) d \lambda(u)\right)\left(\int_{G} \bar{\gamma}(s) d \mu(s)\right) \\
& =\widehat{\chi_{A}}(\gamma) \hat{\mu}(\gamma) .
\end{aligned}
$$


It follows that $\widehat{\mathscr{F}(A})(\gamma)=0$, whenever $\lambda(A)=0$. That is $\widetilde{\mho}(A)=0$ almost everywhere whenever $\lambda(A)=0$. Since $\mathfrak{F}(A)=\chi_{A} * \mu$ is a continuous function, we simply have $\mathfrak{\mho}(A)=0$ whenever $\lambda(A)=0$. It follows that $\mathfrak{F}$ is $\lambda$-continuous. Since for each Borel subset $A$ of $G$,

$$
\mu(A)=\chi-A * \mu(0)=\mathfrak{\Im}(-A)(0)
$$

one sees that $\mu$ is also $\lambda$-continuous.

For the converse, define for each $t \in G$, the set-function $\widetilde{\mho}_{t}: \mathcal{B}(G) \rightarrow X$ by

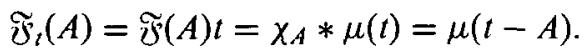

We claim that $\lim _{\lambda(A) \rightarrow 0}\left\|\widetilde{\mho}_{t}(A)\right\|=0$ uniformly in $t$. Indeed fix $\varepsilon>0$. Since by hypothesis $\mu$ is $\lambda$-continuous, there is a $\delta>0$ such that

$$
\lambda(A)<\delta \text { implies }\|\mu(A)\|<\varepsilon .
$$

Let $t$ be in $G$. By the translation invariance property of the Haar measure $\lambda$, one has if $\lambda(A)<\delta$ then $\lambda(t-A)<\delta$, and thus

$$
\left\|\mathfrak{F}_{t}(A)\right\|=\|\mu(t-A)\|<\varepsilon .
$$

That is for every $\varepsilon>0$, there exists $\delta>0$ such that $\sup _{t}\|\mathfrak{F}(A)(t)\|<\varepsilon$, whenever $\lambda(A)<\delta$. This proves our claim, and shows that $\mathfrak{F}$ is absolutely continuous with respect to $\lambda$.

On the other hand, we also have $\|\mathfrak{F}(A)\|_{\infty} \leq\|\mu\|$, for each $A \in \mathcal{B}(G)$. That is

$$
\sup \left\{\|\mathfrak{F}(A)\|_{\infty}, A \in \mathcal{B}(G)\right\}<\infty
$$

This means by [4, Proposition I.1.11], that the measure $\widetilde{F}$ is of bounded semivariation. A corollary of the Carathéodory-Hahn-Kluvanek Extension Theorem (see [4, p. 27-28]) now ensures that $\widetilde{F}$ is strongly additive. Thus by [4, Theorem VI.1.1.], the operator $T_{\mu}$ is weakly compact. This finishes the proof.

Our next result characterizes those convolution operators that are compact on $\mathcal{C}(G)$.

THEOREM 3.3. Let $G$ be a compact metrizable abelian group with Haar measure $\lambda$. Let X be a Banach space, and let $\mu$ be an $X$-valued countably additive measure of bounded variation. Let $T_{\mu}: \mathcal{C}(G) \rightarrow \mathcal{C}(G, X)$ be the convolution operator defined by $T_{\mu}(f)=f * \mu$ for all $f \in \mathcal{C}(G)$. Then the following properties are equivalent:

(a) the operator $T_{\mu}$ is compact;

(b) the measure $\mu$ is absolutely continuous with respect to $\lambda$ and has relatively compact range.

Proof. Suppose the convolution operator $T_{\mu}$ is compact. Then in particular $T_{\mu}$ is weakly compact, and thus by Theorem 3.2, $\mu$ is $\lambda$-continuous. According to Lemma 3.1 , the representing measure $\widetilde{F}$ of $T_{\mu}$ is given by $\mathfrak{F}(A)=\chi_{A} * \mu$. On the other hand, since the operator $T_{\mu}$ is compact, it is a well known result (see for example [4, p. 161]) that the representing 
measure $\mathfrak{\wp}$ has relatively compact range. Thus the set $\left\{\chi_{A} * \mu: A \in \mathcal{B}(G)\right\}$ is relatively compact in $\mathcal{C}(G, X)$. We notice that $\mu(A)=\chi_{-A} * \mu(0)=\mathfrak{F}(-A)(0)$. To finish the proof of the implication (a) $\Rightarrow$ (b) it suffices to notice that the evaluation operator $\delta_{0}: \mathcal{C}(G, X) \rightarrow$ $X ; f \mapsto f(0)$ is a continuous linear operator.

Conversely, suppose that the measure $\mu$ is absolutely continuous with respect to Haar measure $\lambda$ and suppose $\mu$ has relatively compact range. We want to show that the operator $T_{\mu}$ is compact.

Consider the operator $S: \mathcal{C}(G) \rightarrow X$ defined as follows: for each $f \in \mathcal{C}(G)$,

$$
S f=\int_{G} f(s) d \mu(s)
$$

Since the measure $\mu$ has relatively compact range, the operator $S$ is compact [4]. Therefore, its adjoint $S^{*}: X^{*} \rightarrow(\mathcal{C}(G))^{*}$ is also compact. Since for each $x^{*} \in X^{*}$, and for every $f \in \mathcal{C}(G)$, we have

$$
\left\langle S^{*} x^{*}, f\right\rangle=\left\langle x^{*}, S f\right\rangle=x^{*}\left(\int_{G} f(s) d \mu(s)\right)=\int_{G} f(s) d x^{*} \mu(s),
$$

it follows that $S^{*} x^{*}=x^{*} \mu$. Since the measure $\mu$ is absolutely continous with respect to $\lambda$, we see that the operator $S^{*}$ takes its values in $\mathcal{L}^{1}(G)$. Moreover, the compactness of the operator $S^{*}$ implies that the set $K=\left\{x^{*} \mu:\left\|x^{*}\right\| \leq 1\right\}$ is compact in $\mathcal{L}^{1}(G)$.

Now for each $t \in G$ and for every $x^{*} \in X^{*}$, consider the element $x^{*} \otimes \delta_{t}$ of $(\mathcal{C}(G, X))^{*}$ given by $\left\langle x^{*} \otimes \delta_{t}, \varphi\right\rangle=x^{*} \varphi(t)$ for every $\varphi \in \mathcal{C}(G, X)$. Then we observe that for each $f \in \mathcal{C}(G)$,

$$
\left\langle T_{\mu}^{*}\left(x^{*} \otimes \delta_{t}\right), f\right\rangle=\left\langle x^{*} \otimes \delta_{t}, T_{\mu} f\right\rangle=x^{*} f * \mu(t)=\int_{G} f d x^{*} \mu_{t}
$$

where $\mu_{t}$ is the measure defined by $\mu_{t}(A)=\mu(t-A)$. Thus $T_{\mu}^{*}\left(x^{*} \otimes \delta_{t}\right)=x^{*} \mu_{t}$. Since $\mu$ is $\lambda$ continuous, so is the scalar measure $x^{*} \mu$ for each $x^{*} \in X^{*}$ and for every $t \in G$. That is the set $T_{\mu}^{*}(\mathcal{E})$ lies in $\mathcal{L}^{1}(G)$, where $\mathcal{E}=\left\{x^{*} \otimes \delta_{t}:\left\|x^{*}\right\| \leq 1, t \in G\right\}$. We are going to show that in fact, the set $T_{\mu}^{*}(\mathcal{E})$ is compact.

To this end, we first notice that the map

$$
\begin{aligned}
& \phi: G \times \mathcal{L}^{1}(G) \rightarrow \mathcal{L}^{1}(G) \\
&(t, f) \mapsto \bar{f}_{t},
\end{aligned}
$$

where $\bar{f}_{t}(s)=f(t-s)$, is continuous. Indeed if $\left(t_{n}, f_{n}\right)$ converges to $(t, f)$ in $G \times \mathcal{L}^{l}(G)$ then

$$
\begin{aligned}
\left\|\bar{f}_{t}-\bar{f}_{n t_{n}}\right\|_{1} & \leq \int_{G}\left|f(t-u)-f\left(t_{n}-u\right)\right| d \lambda(u) \\
& +\int_{G}\left|f\left(t_{n}-u\right)-f_{n}\left(t_{n}-u\right)\right| d \lambda(u) \\
& =\left\|\bar{f}_{t}-\bar{f}_{t_{n}}\right\|_{1}+\left\|f-f_{n}\right\|_{1} .
\end{aligned}
$$


For every $f \in \mathcal{L}^{1}(G)$, it is easily checked that the map $t \mapsto \bar{f}_{t}$ is continuous (see for example [7]). One then deduces the continuity of $\phi$. It follows that the set $T_{\mu}^{*}(\mathcal{E})$ is compact as the image by $\phi$ of the compact $G \times K$. This proves our claim.

Now for simplicity, let $B$ denote the unit ball of the dual of $\mathcal{C}(G, X)$, and let ext $B$ be the set of its extreme points. Thus we have

$$
\left.T_{\mu}^{*}(B) \subset T_{\mu}^{*} \overline{\overline{c o}(\operatorname{ext} B)}^{\text {weak } *} \subset \overline{\overline{c o}\left(\bar{T}_{\mu}^{*}(\operatorname{ext} B)\right.}\right)^{\text {weak * }} .
$$

It is a well known fact [10] that ext $B$ is contained in $\mathcal{E}$. This implies that $\overline{T_{\mu}^{*}(\text { ext } B)}$ is compact and by Mazur's Theorem (see [4]), so is $\overline{c o}\left(\overline{T_{\mu}^{*}(\operatorname{ext} B)}\right)$. It follows that in particular $\overline{c o}\left(\overline{T_{\mu}^{*}(\operatorname{ext} B)}\right)$ is weakly compact and thus it is weak ${ }^{*}$ closed. Therefore

$$
\overline{\overline{c o}}\left(\overline{T_{\mu}^{*}(\operatorname{ext} B)}\right){ }^{\text {weak * }}=\overline{c o}\left(\overline{T_{\mu}^{*}(\operatorname{ext} B)}\right) .
$$

Accordingly,

$$
T_{\mu}^{*}(B) \subset \overline{c o}\left(\overline{T_{\mu}^{*}(\operatorname{ext} B)}\right) .
$$

This shows that $T_{\mu}^{*}(B)$ has compact closure and hence terminates our proof.

As promised in the introduction, we are now ready to give a characterization of those Banach spaces $X$ where for every $X$-valued measure of bounded variation, the convolution operator $T_{\mu}: \mathcal{C}(G) \rightarrow \mathcal{C}(G, X)$ is compact as soon as it is weakly compact. First let us recall that a Banach space $X$ has the complete continuity property (CRP) if for any $X$-valued measure of bounded variation $\mu$ defined on a group $G$, which is absolutely continuous with respect to Haar measure, the measure $\mu$ has a relatively compact range. For more on the CRP property see [5] or [9]. Our next Corollary gives a complete answer to Question 1.

COROLlaRY 3.4. Let $G$ be a compact metrizable abelian group with Haar measure $\lambda$. A Banach space $X$ has the complete continuity property if and only if for every $X$-valued measure $\mu$ of bounded variation, the convolution operator $T_{\mu}: \mathcal{C}(G) \rightarrow \mathcal{C}(G, X)$ defined by $T_{\mu} f=f * \mu$, for $f \in \mathcal{C}(G)$, is compact whenever it is weakly compact.

4. $p$-summing and nuclear convolution operators on $\mathcal{C}(G)$. In this section, we shall study the vector valued counterpart of Theorem 1.3. More precisely, we shall examine those convolution operators that are either $p$-summing or nuclear. Recall that a bounded linear operator $T$ from a Banach space $X$ into a Banach space $Y$ is said to be $p$-summing $(1 \leq p<\infty)$, if there exists a constant $C>0$ such that for any $x_{1}, x_{2}, \ldots, x_{n}$ in $X$

$$
\left(\sum_{i=1}^{n}\left\|T x_{i}\right\|^{p}\right)^{1 / p} \leq C \sup \left\{\left(\sum_{i=1}^{n}\left|x^{*}\left(x_{i}\right)\right|^{p}\right)^{1 / p}: x^{*} \in X^{*},\left\|x^{*}\right\| \leq 1\right\} .
$$


We denote by $\pi_{p}(T)$ the $p$-summing norm of $T$, which is the smallest constant $C$ such that the inequality (4.1) holds. The operator $T$ is said to be nuclear if there exist sequences $\left\{x_{n}\right\}_{n \geq 1}$ in $X$ and $\left\{x_{n}^{*}\right\}_{n \geq 1}$ in $X^{*}$ such that

$$
T x=\sum_{n=1}^{\infty} x_{n}^{*}(x) x_{n}, \text { for each } x \in X
$$

and

$$
\sum_{n=1}^{\infty}\left\|x_{n}^{*}\right\|\left\|x_{n}\right\|<\infty
$$

For an extensive study of $p$-summing and nuclear operators, we refer the reader to [3]. We first consider the case of the $p$-summing convolution operators for $1 \leq p<\infty$.

First let $1 \leq q \leq \infty$ and $1 / p+1 / q=1$, and let $\mu$ be a vector measure defined on a compact metrizable abelian group $G$ with values in a Banach space $X$. For every measureable set $A \subset G$, let $\mathcal{S}^{p}(A)$ be the set of all the step functions $f=\Sigma_{i} a_{i} \chi_{A_{i}} \in \mathcal{L}^{p}(G)$ where the $A_{i}$ are disjoint Borel subsets of $G$ contained in $A$, and such that $\|f\|_{p} \leq 1$. For a Borel subset of $G$, the $q$-semivariation of $\mu$ at $A$ is defined as follows:

$$
\tilde{\mu}_{q}(A)=\sup _{\Sigma_{i} a_{i} X_{A_{i}} \in \mathcal{S}^{p}(A)}\left\|\sum a_{i} \mu\left(A_{i}\right)\right\|_{X} .
$$

A vector measure $\mu$ valued in a Banach space $X$ is said to be of bounded q-semivariation if $\tilde{\mu}_{q}(G)<\infty$, and the measure is said to be of bounded average range if there exists a constant $C>0$ such that $\|\mu(A)\| \leq C \lambda(A)$, for each Borel subset $A$ of $G$.

The following fact is well known [2] and will be used later.

REMARK 4.1. A vector measure $\mu$ is of bounded average range if and only if it is of bounded $\infty$-semivariation, i.e. $\tilde{\mu}_{\infty}(G)<\infty$.

Proof. Clearly, we have

$$
\sup _{A \in \mathcal{B}(G)} \frac{\|\mu(A)\|}{\lambda(A)} \leq \sup _{\Sigma_{i} a_{i} \lambda_{A_{i}} \in \mathcal{S}^{1}(G)}\left\|\sum a_{i} \mu\left(A_{i}\right)\right\|=\tilde{\mu}_{\infty}(G) .
$$

So if $\tilde{\mu}_{\infty}(G)<\infty$, then $\|\mu(A)\| \leq \tilde{\mu}_{\infty}(G) \lambda(A)$ for each Borel subset $A$ of $G$.

Conversely, if $f=\Sigma_{i} a_{i} \chi_{A_{i}} \in \mathcal{S}^{1}(G)$, then since the $A_{i}$ are disjoint Borel subsets of $G$ we notice that $\|f\|_{1}=\left\|\Sigma\left|a_{i}\right| \chi_{A_{i}}\right\|_{1}$, and therefore

$$
\begin{aligned}
\left\|\Sigma a_{i} \mu\left(A_{i}\right)\right\| & \leq \Sigma\left|a_{i}\right|\left\|\mu\left(A_{i}\right)\right\| \\
& =\Sigma\left|a_{i}\right| \frac{\left\|\mu\left(A_{i}\right)\right\|}{\lambda\left(A_{i}\right)} \lambda\left(A_{i}\right) \\
& =\int_{G} \Sigma\left|a_{i}\right| \chi_{A_{i}} \Sigma \chi_{A_{j}} \frac{\left\|\mu\left(A_{j}\right)\right\|}{\lambda\left(A_{j}\right)} d \lambda \\
& \leq\left\|\Sigma\left|a_{i}\right| \chi_{A_{i}}\right\|_{1}\left\|\Sigma \chi_{A_{i}} \frac{\left\|\mu\left(A_{i}\right)\right\|}{\lambda\left(A_{i}\right)}\right\|_{\infty} \\
& \leq\|f\|_{1} \sup _{B \subset G} \frac{\|\mu(B)\|}{\lambda(B)} .
\end{aligned}
$$


This of course shows that $\tilde{\mu}_{\infty}(G)<\infty$ if $\mu$ is of bounded average range.

The reader is referred to [2] for the following result.

THEOREM 4.2. Let $G$ be a compact metrizable abelian group with Haar measure $\lambda$. Let $1 \leq p \leq \infty$ and $1 / p+1 / q=1$. There is an isomorphism between the set of all bounded linear mappings $U: \mathcal{L}^{p}(G) \rightarrow X$ and the set of all the measures $v: \mathcal{B}(G) \rightarrow X$ of bounded $q$-semivariation given by the equality

$$
U(f)=\int_{G} f d v, \text { for } f \in \mathcal{L}^{p}(G)
$$

and in particular

$$
v(A)=U\left(\chi_{A}\right),
$$

for every Borel subset $A$ of $G$. Moreover, if $U$ and $v$ are in such correspondence, then $\|U\|=\tilde{v}_{q}(G)$.

Before stating our next result, we shall include for the sake of completeness the following well known result (see [3, p. 56] for a more general statement).

Lemma 4.3. Let $G$ be a compact metrizable abelian group. Let $X$ be a Banach space, and let $\mu$ be an $X$-valued measure of bounded variation. Let $T_{\mu}: \mathcal{C}(G) \rightarrow \mathcal{C}(G, X)$ be the convolution operator defined by $T_{\mu}(f)=f * \mu$ for all $f \in \mathcal{C}(G)$. Suppose that $T_{\mu}$ is p-summing, for $1 \leq p<\infty$. Then $T_{\mu}$ factors through $\mathcal{L}^{p}(G)$.

Proof. First for every $f \in \mathcal{C}(G)$, consider $f_{-t}(y)=f(y+t)$. Then clearly $f_{-t} \in \mathcal{C}(G)$ and a simple calculation shows that

$$
\left\|f_{-t} * \mu\right\|=\|f * \mu\|
$$

Now suppose $T_{\mu}$ is $p$-summing. One can find a non-negative Pietsch measure [3, p. 48] $\sigma$ on the group $G$ such that

$$
\left\|T_{\mu} f\right\|^{p} \leq\left(\pi_{p}\left(T_{\mu}\right)\right)^{p} \int_{G}|f(s)|^{p} d \sigma(s)
$$

Here $\pi_{p}\left(T_{\mu}\right)$ denotes the $p$-summing norm of the operator $T_{\mu}$. Define $\sigma_{t} \in \mathcal{C}(G)^{*}$ by

$$
\left\langle\sigma_{t}, f\right\rangle=\left\langle\sigma, f_{-t}\right\rangle
$$

for all $f \in \mathcal{C}(G)$ and for every $t \in G$. It then follows from (4.2) that

$$
\left\|T_{\mu}(f)\right\|^{p}=\left\|T_{\mu}\left(f_{-t}\right)\right\|^{p} \leq \pi_{p}^{p}\left(T_{\mu}\right) \int_{G}\left|f_{-t}(s)\right|^{p} d \sigma(s)=\pi_{p}^{p}\left(T_{\mu}\right) \int_{G}|f(s)|^{p} d \sigma_{t}(s) .
$$

This shows that $\sigma_{t}$ is also a Pietsch measure for the operator $T_{\mu}$. It is easy to show that the map $G \rightarrow \mathcal{C}(G)^{*}: t \mapsto \sigma_{t}$ is continuous when $\mathcal{C}(G)^{*}$ is endowed with the weak ${ }^{*}$ topology. Thus 

the weak ${ }^{*}$ integral $\int_{G} \sigma_{t} d \lambda(t)$ exists. Therefore there exists a measure $\nu \in \mathcal{C}(G)^{*}$ such that for
every $f \in \mathcal{C}(G)$

$$
\langle\nu, f\rangle=\int_{G}\left\langle\sigma_{t}, f\right\rangle d \lambda(t) .
$$

Thus $\nu$ is a non-negative regular Borel measure and since

$$
v(G)=\int_{G} \sigma_{t}(G) d \lambda(t)=\int_{G} \chi_{G} d \lambda=1,
$$

we see that $\nu$ is a probability measure. Thus by integrating both sides of (4.3) one has, for each $f \in \mathcal{C}(G)$,

$$
\left\|T_{\mu} f\right\|^{p} \leq \pi_{p}^{p}\left(T_{\mu}\right) \int_{G}\left\langle\sigma_{t},|f|^{p}\right\rangle d \lambda(t)=\pi_{p}^{p}\left(T_{\mu}\right)\left\langle\nu,|f|^{p}\right\rangle .
$$

This shows that $v$ is also a Pietsch measure for $T_{\mu}$. To finish the proof we only need to show that $v$ is translation invariant, and so must coincide with the Haar measure $\lambda$ on $G$. To this end, note that for any $t_{0} \in G$ and $f \in \mathcal{C}(G)$, if we use Fubini's theorem and the translation invariance property of the Haar measure we have

$$
\begin{aligned}
\left\langle\nu, f_{-t_{0}}\right\rangle & =\int_{G}\left\langle\sigma_{t}, f_{-t_{0}}\right\rangle d \lambda(t)=\iint_{G} f_{-t_{0}}(s) d \sigma_{t}(s) d \lambda(t) \\
& =\int_{G} \int_{G} f\left(s+t_{0}\right) d \sigma_{t}(s) d \lambda(t)=\iint_{G} f\left(s+t_{0}+t\right) d \sigma(s) d \lambda(t) \\
& =\iint_{G} f\left(s+t_{0}+t\right) d \lambda(t) d \sigma(s)=\iint_{G} f(s+t) d \lambda(t) d \sigma(s) \\
& =\int_{G} \int_{G} f(s+t) d \sigma(s) d \lambda(t)=\iint_{G} f(s) d \sigma_{t}(s) d \lambda(t) \\
& =\int_{G}\left\langle\sigma_{t}, f\right\rangle d \lambda(t)=\langle\nu, f\rangle .
\end{aligned}
$$

We now state our next result which characterizes those convolution operators which are $p$ summing on $\mathcal{C}(G)$.

THEOREM 4.4. Let $G$ be a compact metrizable abelian group with Haar measure $\lambda$. Let $X$ be a Banach space, and let $\mu$ be an $X$-valued measure of bounded variation. Let $T_{\mu}: \mathcal{C}(G) \rightarrow \mathcal{C}(G, X)$ be the convolution operator defined by $T_{\mu}(f)=f * \mu$ for all $f \in \mathcal{C}(G)$. Let $1 \leq p<\infty$ and $1 / p+1 / q=1$. Then the following properties are equivalent:

(a) the operator $T_{\mu}$ is p-summing;

(b) the measure $\mu$ is of bounded q-semivariation. 
Proof. Suppose the convolution operator $T_{\mu}$ is $p$-summing. By Lemma 4.3 there exists a bounded linear map $U: \mathcal{L}^{p}(G) \rightarrow \mathcal{C}(G, X)$ such that $U j_{p}=T_{\mu}$ where $j_{p}: \mathcal{C}(G) \rightarrow \mathcal{L}^{p}(G)$ is the canonical injection. Let $\delta_{0}: \mathcal{C}(G, X) \rightarrow X$ denote the evaluation operator defined by $\delta_{0}(f)=f(0)$. Consider the operator

$$
\begin{array}{r}
U_{0}=\delta_{0} U: \mathcal{L}^{p}(G) \rightarrow X \\
f \mapsto U f(0) .
\end{array}
$$

Let $\nu$ be the measure of bounded $q$-semivariation associated to the operator $U_{0}$. By Theorem 4.2 we have $U_{0}(f)=\int_{G} f d v$ for every $f \in \mathcal{L}^{p}(G)$. We then notice that for every $f \in \mathcal{C}(G)$,

$$
\int_{G} f(t) d v(t)=U_{0}(f)=U(f)(0)=f * \mu(0)=\int_{G} f(-t) d \mu(t) .
$$

That is $\mu=v^{\prime}$, where $\nu^{\prime}(A)=v(-A)$. This shows that $\mu$ is of bounded $q$-semivariation.

Conversely suppose the measure $\mu$ is of bounded $q$-semivariation. Let $U: \mathcal{L}^{p}(G) \rightarrow X$ be the bounded linear operator associated to $\mu$ as in Theorem 4.2. Then $U(f)=\int_{G} f d \mu$, for
$f \in \mathcal{L}^{p}(G)$. One has

$$
\|U(f)\|=\left\|\int_{G} f d \mu\right\| \leq\|f\|_{p} \tilde{\mu}_{q}(G)=\|f\|_{p}\|U\| .
$$

It follows that for every $f \in \mathcal{L}^{p}(G)$, and for every $t$ and $t^{\prime}$ in $G$

$$
\left\|f * \mu(t)-f * \mu\left(t^{\prime}\right)\right\|_{X}=\left\|\int_{G} f(t-s)-f\left(t^{\prime}-s\right) d \mu(s)\right\|_{X} \leq\left\|\bar{f}_{t}-\bar{f}_{t^{\prime}}\right\|_{p}\|U\|
$$

where $\bar{f}(s)=f(-s)$ for every $s \in G$. This shows that the convolution operator $U_{\mu}: \mathcal{L}^{p}(G) \rightarrow$ $\mathcal{L}^{p}(G, X) ; f \mapsto f * \mu$ actually is a bounded operator into $\mathcal{C}(G, X)$, and that $\left\|U_{\mu}\right\| \leq\|U\|$. It is plain that the following diagram commutes:

$$
\begin{array}{lll}
\mathcal{C}(G) & \stackrel{T_{\mu}}{\longrightarrow} & \mathcal{C}(G, X) \\
j_{p} \searrow & & \nearrow U_{\mu} \\
& \mathcal{L}^{p}(G) &
\end{array}
$$

where $j_{p}$ is the canonical injection. This diagram clearly shows that the operator $T_{\mu}$ is $p$ summing, and completes the proof.

In view of Remark 4.1, we have the following result.

Corollary 4.5. Let $G$ be a compact metrizable abelian group with Haar measure $\lambda$. Let $X$ be a Banach space, and let $\mu$ be an $X$-valued measure of bounded variation. Let $T_{\mu}: \mathcal{C}(G) \rightarrow \mathcal{C}(G, X)$ be the convolution operator defined by $T_{\mu}(f)=f * \mu$ for all $f \in \mathcal{C}(G)$. Then the following properties are equivalent:

(a) the operator $T_{\mu}$ is 1-summing;

(b) the measure $\mu$ is of bounded average range. 
REMARK 4.6. It follows from Theorem 4.4 and Corollary 4.5 that if $X$ is a Banach space that has the Radon-Nikodym property (RNP), and $\mu$ is an $X$-valued measure of bounded variation, then $T_{\mu}: \mathcal{C}(G) \rightarrow \mathcal{C}(G, X)$ is $p$-summing $(1 \leq p<\infty)$ iff there exists $\phi \in \mathcal{L}^{p}(G, X)$ such that $\mu=\phi \cdot \lambda$, in particular $T_{\mu}$ is 1 -summing iff $\mu=\phi \cdot \lambda$ for some $\phi \in \mathcal{L}^{\infty}(G, X)$.

We finish this section by studying those convolution operators that are nuclear on the space of continuous functions $\mathcal{C}(G)$.

THEOREM 4.7. Let $G$ be a compact metrizable abelian group with Haar measure $\lambda$. Let $X$ be a Banach space, and let $\mu$ be an $X$-valued measure of bounded variation. Let $T_{\mu}: \mathcal{C}(G) \rightarrow \mathcal{C}(G, X)$ be the convolution operator defined by $T_{\mu}(f)=f * \mu$ for all $f \in \mathcal{C}(G)$. Then the following properties are equivalent:

(a) the operator $T_{\mu}$ is nuclear;

(b) there exists $h \in \mathcal{C}(G, X)$ such that $\mu=h \cdot \lambda$.

Proof. Suppose the operator $T_{\mu}$ is nuclear. Let $\widetilde{F}$ be the representing measure of $T_{\mu}$. Then $\mathfrak{F}$ is a countably additive $\mathcal{C}(G, X)$-valued measure of bounded variation and there exists $g \in \mathcal{L}^{1}(G,|\mathfrak{F}|, \mathcal{C}(G, X)$ ) such that $\mathfrak{F}=g \cdot|\mathfrak{F}|$ (see for example [2, p. 173]). Here $\mathcal{L}^{l}(G,|\widetilde{F}|, \mathcal{C}(G, X))$ stands for (all classes of) $|\mathfrak{\mho}|$-Bochner integrable functions on $G$ with values in $\mathcal{C}(G, X)$. Moreover, one should note that the function $g$ is actually an essentially bounded function.

Since the operator $T_{\mu}$ is in particular 1-summing, by Corollary 4.5 , the measure $\mu$ is of bounded average range. It then follows that for every $t \in G$, and each Borel subset $A$ of $G$ and for some constant $C>0$,

$$
\|\widetilde{\Im}(A)(t)\|=\left\|\chi_{A} * \mu(t)\right\|=\|\mu(t-A)\| \leq C \lambda(A) .
$$

Thus since the measure $\mathfrak{F}$ takes its values in $\mathcal{C}(G, X)$, one deduces

$$
\|\Im(A)\|_{\infty} \leq C \lambda(A) .
$$

This shows that $\mathfrak{F}$ is of bounded average range and its variation satisfies $|\mathfrak{F}| \leq C \lambda$. Therefore $\widetilde{F}=\phi . \lambda$ for some $\phi \in \mathcal{L}^{\infty}(G, \mathcal{C}(G, X))$. The function $\phi$ can be considered as function of two variables as follows

$$
\phi(u, v)=\phi(u)(v), \text { for each }(u, v) \in G \times G
$$

As in the proof of Theorem 2.1, we define $h \in \mathcal{L}^{1}(G, X)$ by

$$
h(t)=\int_{G} \phi(u-t, u) d \lambda(u) .
$$

We claim that $h$ is a continuous function. To see this, we notice that

$$
\begin{aligned}
h(s) & =\int_{G} \phi(u-s, u) d \lambda(u) \\
& =\int_{G} \phi(v, v+s) d \lambda(v) .
\end{aligned}
$$


Therefore,

$$
\begin{aligned}
\|h(t)-h(s)\| & =\left\|\int_{G} \phi(v, v+t)-\phi(v, v+s) d \lambda(v)\right\| \\
& \leq \int_{G}\|\phi(v, v+t)-\phi(v, v,+s)\| d \lambda(v) .
\end{aligned}
$$

Since the function $t \mapsto \phi(v, v+t)$ is continuous for each $v \in G$, the integrand in the last integral converges to zero as $t$ tends to $s$, for each $v \in G$. On the other hand, the following inequality clearly holds

$$
\|\phi(v, v+t)-\phi(v, v+s)\| \leq 2\|\phi\|_{\infty}
$$

An appeal to the Lebesgue Dominated Convergence Theorem now establishes our claim. The same reasoning as in (2.1) and (2.2) now shows that $\mu=h \cdot \lambda$.

Conversely, suppose $\mu=h \cdot \lambda$ where $h \in \mathcal{C}(G, X)$. Consider the map $g: G \rightarrow \mathcal{C}(G, X)$ : $y \mapsto h_{y}$. Then clearly the function $g$ is continuous and therefore it is $\lambda$-Bochner integrable. Now fix $\varepsilon>0$. According to Lemma 3 of $([4, \mathrm{p} .173])$, there is a sequence $\left(\phi_{n}\right)$ in $\mathcal{C}(G, X)$ and a sequence of Borel sets $\left(E_{n}\right)$ such that

$$
g=\sum_{n=1}^{\infty} \phi_{n} \chi E_{n}
$$

$\lambda$-almost everywhere, and

$$
\int_{G}\|g\| d \lambda \leq \sum_{n=1}^{\infty}\left\|\phi_{n}\right\| \lambda\left(E_{n}\right) \leq \int_{G}\|g\| d \lambda+\varepsilon .
$$

For $f \in \mathcal{C}(G)$, we have

$$
\begin{aligned}
T_{\mu}(f)=f * h & =\int_{G} f(y) \sum_{n=1}^{\infty} \phi_{n} \chi_{E_{n}}(y) d \lambda(y) \\
& =\sum_{n=1}^{\infty} \int_{E_{n}} f(y) d \lambda(y) \phi_{n} .
\end{aligned}
$$

Obviously, the map $f \mapsto L_{n}(f)=\int_{E_{n}} f d \lambda$ is a bounded linear functional on $\mathcal{C}(G)$ and $T_{\mu}(f)=\sum_{n=1}^{\infty} L_{n}(f) \phi_{n}$ and

$$
\sum_{n=1}^{\infty}\left\|L_{n}(f)\right\|\left\|\phi_{n}\right\| \leq \int_{G}\|g\| d \lambda+\varepsilon
$$

This proves that $T_{\mu}$ is nuclear and ends our proof. 
REFERENCES

1. A. V. Bukhvalov and A. A. Danielevich, Boundary properties of analytic and harmonic functions with values in Banach space, Mat. Zametki, 31, (1982), 203-214.

2. N. Dinculeanu, Vector measures (Pergamon Press, 1967).

3. J. Diestel, H. Jarchow and A. Tonge, Absolutely summing operators, Cambridge studies in advanced mathematics $\mathbf{4 3}$ (Cambridge University Press, 1995).

4. J. Diestel and J. J. Uhl, Jr, Vector measures, Math. Surveys, 15 (AMS, Providence, RI, 1977).

5. M. K. Girardi, Ph.D. Thesis (University of Illinois, 1990).

6. E. Hewitt and K. A. Ross, Abstract harmonic analysis I-II (Springer-Verlag, 1970).

7. Y. Katznelson, An introduction to harmonic analysis (Dover Publication Inc, New York, 1976).

8. F. Lust-Piquard, Properties geometriques des sous-espaces invariants par translation de $\mathrm{L}^{\prime}(G)$ et $\mathrm{C}(G)$, Seminaire sur la geometrie des espaces de Banach, Ecole Polytechnique, 26, (1977-78).

9. K. Musial, Martingales of Pettis integrable functions, in Measure theory, Oberwolfach, 1979. Lecture Notes in Mathematics 794, (Springer-Verlag, 1980).

10. I. Singer, Sur la meilleure approximation des fonctions abstraites continues a valeurs dans un espace de Banach, Rev. Roumaine Math. Pures Appl., 2, (1957), 245-262.

11. P. Wojtaszczyk, Banach spaces for analysts (Cambridge University Press, 1991).

Mangatiana A. Robdera

William Paterson College

300 POMPTON RD.

WAYNE, NJ 07470

E-mail: robdera@frontier.wilpaterson.edu
Paulette Saab

Department of Mathematics

UNIVERSITY OF MisSOURI

Columbia, MO 65211

E-mail: paula@math.missouri.edu

Present address

Eastern Mediterranean University

GaZimaguZa

Via Mersin 10

TURKEY

E-mail: robdera@mozart.emu.edu.tr 